\title{
Research on Pulse Laser for Simulating Transient Radiation Effects on MOS Devices
}

\author{
Li Mo $^{1 \mathrm{a}}$, Sun Peng ${ }^{1 \mathrm{~b}}$, Li Linzhe ${ }^{2}$, Li Jie ${ }^{2}$, Qi Chunhua ${ }^{2}$, Xiao Liyi ${ }^{2 c^{*}}$ \\ ${ }^{1}$ Microsystem \& Terahertz Research Center of China Academy of Engineering Physics, Chengdu, \\ 610200, China \\ ${ }^{2}$ Microelectronics Center of Harbin Institute of Technology, Harbin, 150001, China \\ a, b email: limo, sunpeng@mtrc.ac.cn, ${ }^{c^{\star}}$ email: xiaoly@hit.edu.cn
}

Keywords: MOS; Pulse Laser; Transient Radiation Effects; TCAD

\begin{abstract}
In this paper, the transient radiation effect of MOS devices irradiated by pulse laser is studied using TCAD tool. Firstly, the laser radiation model is built up based on the TCAD tool, and also MOS devices model are created and the I-V characteristics are simulated for proving their correctness. With the pulse laser model, the transient responses of MOS devices working in different modes irradiated by pulse laser are simulated. The results show that the transient radiation effect caused by pulse laser when irradiating MOS devices leads to leakage current of the substrate of MOS devices, and the current amplitude increases with the laser's intensity.
\end{abstract}

\section{Introduction}

In the radiation environment, the interaction of gamma rays, neutrons etc. with semiconductor devices can induce ionization effects and short term effects, which can severely impact the function of devices and circuits [1]. Therefore, devices and circuits working in harsh environments have to be tested before using them in certain missions. Pulsed X-ray machines and linear accelerators are commonly used to study these radiation effects and analysis the reliability of devices and circuits; however, they require relatively large installations and have a high cost of operation.

The laser interacting with semiconductor materials can produce photoelectric effect, which can excite electron-hole pairs in the semiconductor. This is similar with the radiation effect caused by gamma or $\mathrm{X}$ rays and ions that can inject excess carries to the semiconductor materials or devices. Habing from SNL first reported the use of pulse laser to simulate the radiation effects of semiconductor devices. It is proved that the pulse laser can effectively simulate the transient radiation effect [2]. In practical use, the pulse laser facility has the advantages of low cost, easy control and precise spatial resolution, which make the laser simulation technique very popular in radiation area. It can simulate the radiation effect and help analysis the reliability of semiconductor devices flexibly and safely in the laboratory [3-6].

TCAD (Technology Computer Aided Design) tool can be used to model semiconductor fabrication and semiconductor device operation. It can help study the physical characteristics of devices used in circuits from lower level. In this paper, based on the TCAD tool, a laser radiation model is built up for deeply studying the transient radiation effect of the pulse laser interacting with MOS (Metal Oxide Semiconductor) devices. The structure of this paper is as follows: in the first section, the laser radiation model based on TCAD is descripted; besides, the MOS devices used for simulation is created. Then, with the laser radiation model, simulation results of pulse laser irradiating MOS devices are given and analyzed. And finally the paper draws conclusions.

\section{Simulation Setup}

\section{A. Pulse laser modeling}

As shown in Figure 1, when a laser illuminates a semiconductor structure, a reflection/ transmission process occurs at interfaces. The photons transmitted inside the semiconductor device are absorbed by the semiconductor material or escape from the structure. If the energy of the 
absorbed photons exceeds the band gap of the semiconductor material, the electron-hole pairs are excited by the absorbed photons. TCAD setup for the laser radiation model to solve the event as described above needs three basic models: Complex Refractive Index Models, Optical Solver, and Optical Generation Model. The refractive index and the extinction coefficient can be specified using different Complex Refractive Index Models, such as constant, wavelength dependent, temperature dependent, carrier dependent; the Optical Solver is used to calculate the absorbed photon density; the Optical Generation Model needs a suitable quantum yield model to define how the absorbed photons are converted into electron-hole pairs. What's more, the Excitation Model need to be specified to define everything concerning illumination including laser illuminating direction, polarization, illumination window and laser parameters such as laser wavelength, intensity, pulse width and pulse shape. In this paper, the laser with wavelength of $1064 \mathrm{~nm}$ is chosen which has been proven the optimum choice for testing silicon ICs owing to the high ionization uniformity with depth [7, 8]. The pulse width of laser is set to 15 ns (illuminating devices from 30 ns to $45 \mathrm{~ns}$ ), and the laser intensity ranges from $1 \mathrm{e} 1 \mathrm{~W} / \mathrm{cm}^{2}$ to $1 \mathrm{e} 6 \mathrm{~W} / \mathrm{cm}^{2}$. The laser illuminating direction is along with $\mathrm{Y}$ axis direction, and illumination window covers the whole devices as shown in Figure 2.

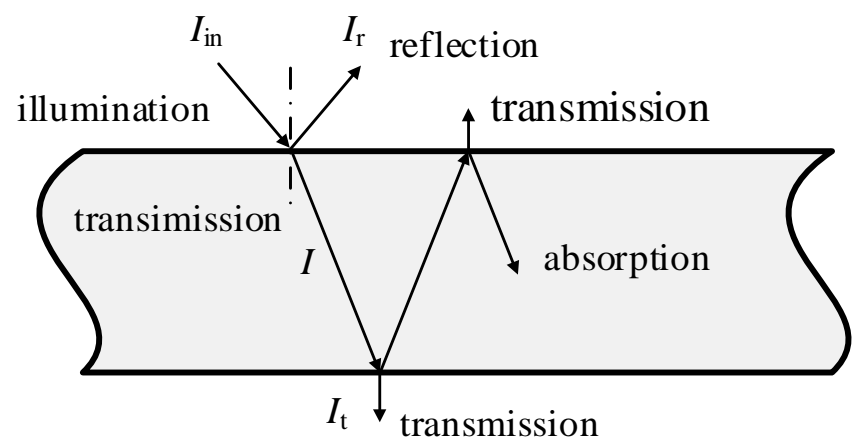

Fig.1. laser illumination model

\section{B. MOS device structure}

We created two typical MOS devices model using TCAD: NMOS (Negative MOS) and PMOS (Positive MOS), and the 2D structure is as shown in Figure 2. The differences between NMOS and PMOS are the different doping types and levels in different regions. There are four contacts named source, gate, drain and substrate. The length of MOS devices channel are $45 \mathrm{~nm}$. The characteristics for input and output of NMOS and PMOS devices were simulated, and the characteristics curves were shown in Figure 3 and Figure 4 respectively.

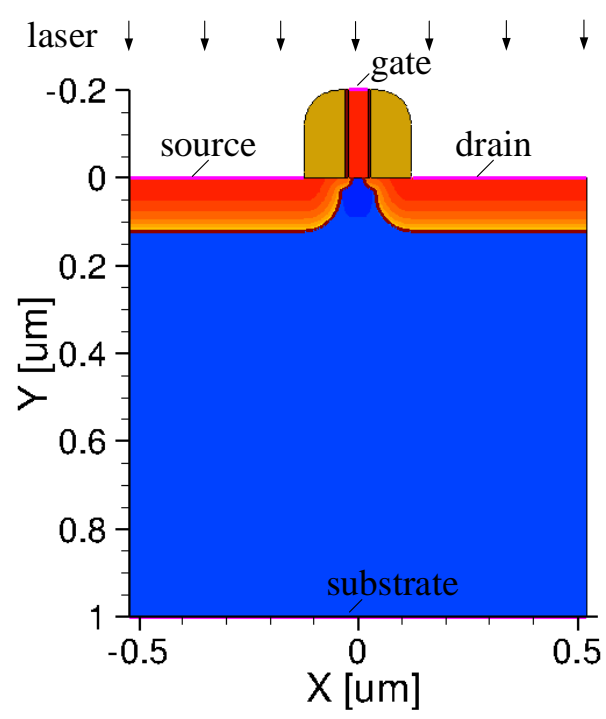

Fig.2. MOS 2D TCAD structure 


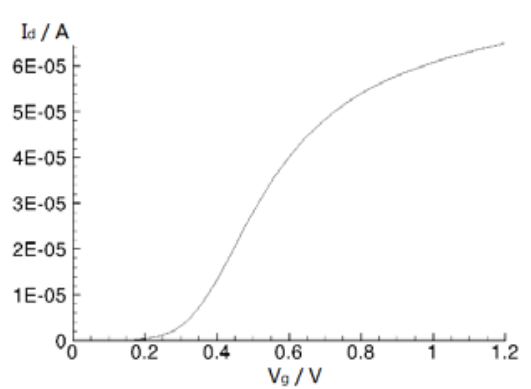

(a) $\mathrm{I}_{\mathrm{d}}-\mathrm{V}_{\mathrm{g}}$ curve

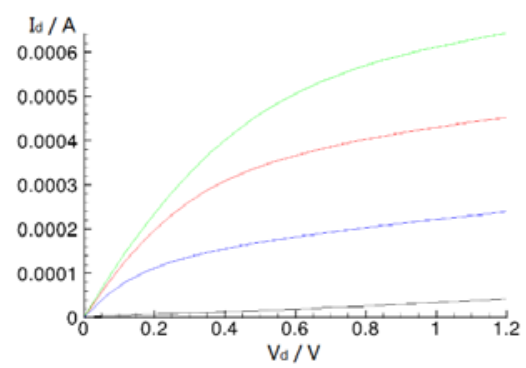

(b) Id- $V_{d}$ curves

Fig.3. NMOS input and output characteristics curves

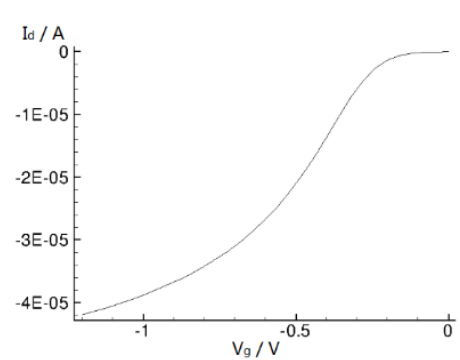

(a) $\mathrm{I}_{\mathrm{d}}-\mathrm{V}_{\mathrm{g}}$ curve

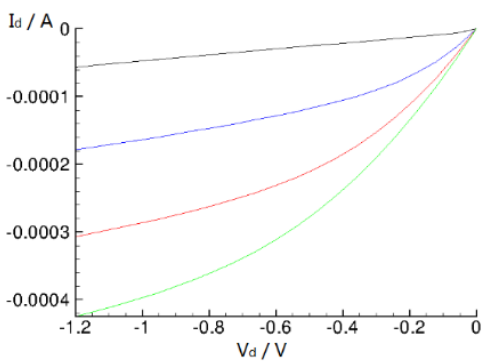

(b) Id- $\mathrm{V}_{\mathrm{d}}$ curves

Fig.4. PMOS input and output characteristics curves

\section{Simulation Results and Analysis}

In digital circuits, the MOS devices usually work on two modes: on-mode and off-mode. As for NMOS, when the gate voltage is high, it works on on-mode, and when the gate voltage is low, it works on off-mode. On the contrary, as for PMOS, high gate voltage means off-mode operating, and low gate voltage means on-mode operating. When the MOS devices work on different modes, their contacts voltage are different. So we set different bias voltage on contacts to simulate MOS devices working on different modes. The bias voltages of MOS devices' different contacts as well as their work mode are listed in Table 1.

Table 1 Contacts bias voltages of MOS devices

\begin{tabular}{|c|c|c|c|c|c|}
\hline MOS device & drain voltage/V & gate voltage/V & source voltage/V & substrate voltage/V & work mode \\
\hline \multirow{2}{*}{ NMOS } & 0 & 1 & 0 & 0 & on \\
\cline { 2 - 6 } & 1 & 0 & 0 & 0 & off \\
\hline \multirow{2}{*}{ PMOS } & 1 & 0 & 1 & 1 & on \\
\cline { 2 - 6 } & 0 & 1 & 1 & 1 & off \\
\hline
\end{tabular}

Figure 5 and Figure 6 illustrated the transient response of the drain, source, and substrate currents when the laser with intensity of $1 \mathrm{e} 4 \mathrm{~W} / \mathrm{cm}^{2}$ irradiated NMOS worked on on-mode and off-mode; Figure 7 and Figure 8 illustrated the PMOS's transient response. And in Figure 9, we demonstrated the relationship between laser intensity and photocurrent of different contacts for NMOS work on different modes.
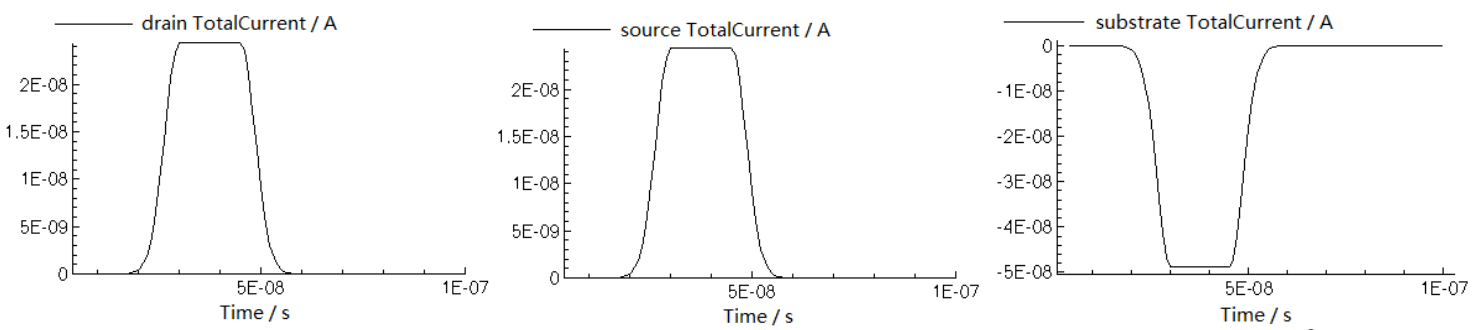

Fig.5.on-mode NMOS transient response (laser intensity $=1 \mathrm{e} 4 \mathrm{~W} / \mathrm{cm}^{2}$ ) 

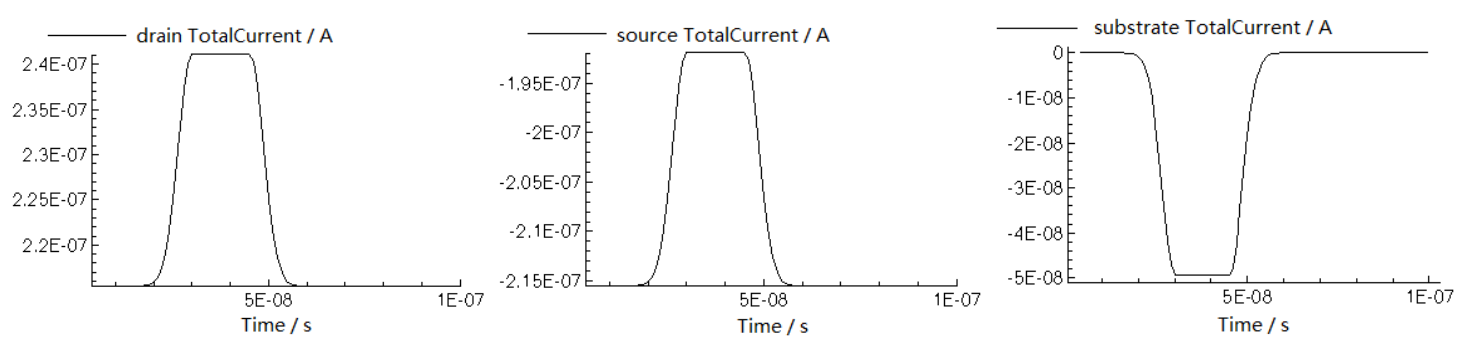

Fig.6.off-mode NMOS transient response (laser intensity $=1 \mathrm{e} 4 \mathrm{~W} / \mathrm{cm}^{2}$ )
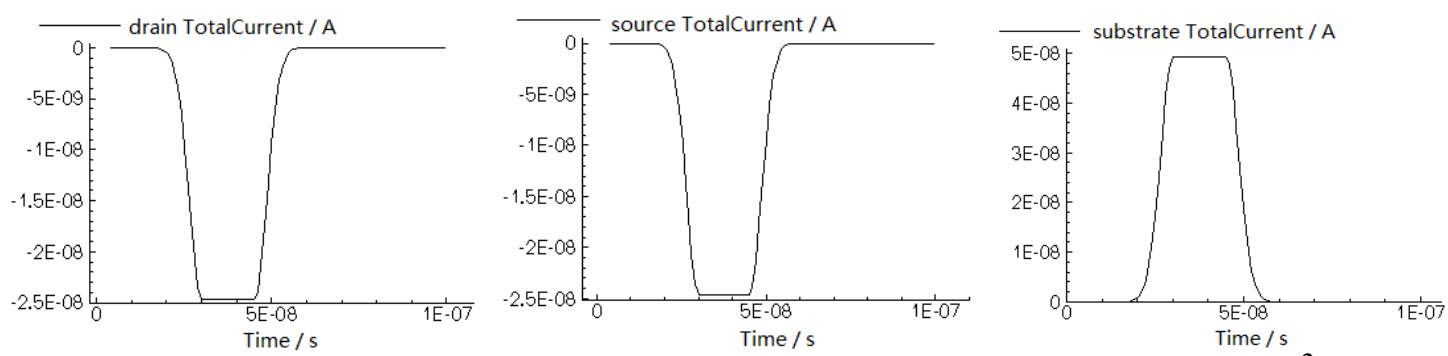

Fig.7.on-mode PMOS transient response (laser intensity $=1 \mathrm{e} 4 \mathrm{~W} / \mathrm{cm}^{2}$ )
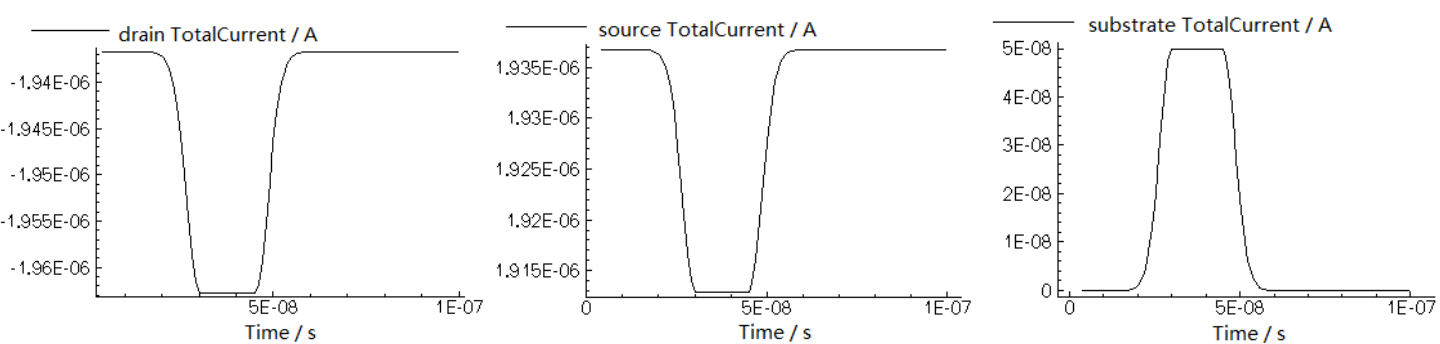

Fig.8.off-mode PMOS transient response (laser intensity $=1 \mathrm{e} 4 \mathrm{~W} / \mathrm{cm}^{2}$ )

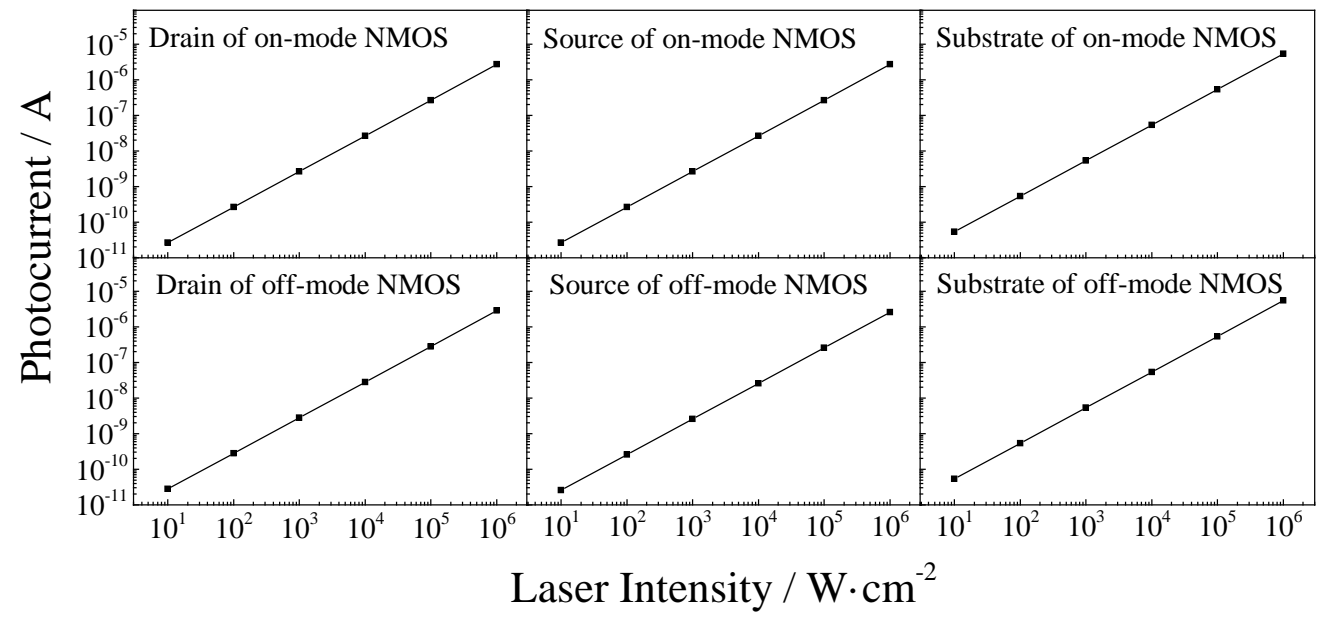

Fig.9. photocurrent versus laser intensity for NMOS

When MOS devices were irradiated by pulse laser, the current of source, drain and substrate contacts changed. The transient responses were different for different devices work on different modes. In case of working on on-mode, under the action of source-substrate PN junction and drain-substrate PN junction, the photocurrent flowed from source and drain to substrate as for NMOS or from substrate to source and drain as for PMOS. In case of working on off-mode, if there was no laser radiation, only the leakage current between drain and source existed and the substrate current was close to 0 ; when the devices were irradiated by pulse laser, the drain current increased and the source current decreased, and the substrate current appeared.

Transient radiation effect occurred when pulse laser irradiating the MOS devices no matter which mode they worked on, and it led to the changes of different contacts' total current due to the photocurrent caused by the laser radiation. As illustrated in Figure 9, the photocurrent amplitude increased with the pulse laser's intensity. 


\section{Conclusion}

In this paper, the laser radiation model has been descripted, and the laser radiation simulation was able to be implemented with this model using TCAD tool. Simulation results obtained using the laser radiation model to simulate the transient radiation effect with MOS devices have been presented and analyzed. The results demonstrated that the transient radiation effect caused by pulse laser led to leakage current of the substrate of MOS devices no matter which mode they worked on, and the current amplitude increased with the pulse laser's intensity. This could cause faults for circuits if the current amplitude was sufficiently high.

\section{Acknowledgement}

This work is conducted under the support of President Funding of CAEP (2014-1- 100).

\section{References}

[1] Deval Y, Lapuyade H, Fouillat P, et al. Evaluation of a design methodology dedicated to dose rate hardened linear integrated circuits[J]. IEEE Transactions on Nuclear Science, 2001, 49(3):237-242.

[2] Habing D H. The use of lasers to simulate radiation-induced transients in semiconductor devices and circuits[J]. IEEE Trans. Nucl. Sci., 1965,12(5):91-100.

[3] Nation S A, Massengill L W, Mcmorrow D, et al. Laser Dose-Rate Simulation to Complement LINAC Discrete Device Data[J]. IEEE Transactions on Nuclear Science, 2009, 55(6):3114-3121.

[4] López-Calle, I, Franco, F.J, Agapito, J.A, et al. LASER system for space environment emulation[C]// Electron Devices (CDE), 2011 Spanish Conference on. IEEE, 2011:1-4.

[5] Daniel C, Plettner C, Schuttauf A, et al. Laser Pulse Tests of Bipolar Junction Transistors (BJTs) for SET Analysis[C]// Radiation Effects Data Workshop. IEEE, 2014.

[6] Liang T, Bauer J, Cimeno M, et al. Measurements of High-Intensity Laser Induced Ionizing Radiation at SLAC[J]. Shielding Aspects of Accelerators, Targets and Irradiation Facilities SATIF-12 Workshop Proceedings. pp.40-53, 2015.

[7] Nikiforov A Y, Skorobogatov P K. "Physical principles of laser simulation for the transient radiation response of semiconductor structures, active circuit elements, and circuits: A linear model”. Russian Microelectronics, vol 33, pp. 68-79, 2004.

[8] Nikiforov A Y, Skorobogatov P K, Egorov A N, et al. "Selection of optimal parameters of laser radiation for simulating ionization effects in silicon bulk-technology microcircuits." Russian Microelectronics, vol 43, pp. 133-138, 2014. 